\title{
INTEGRAL DOMAINS WITH FINITELY GENERATED GROUPS OF DIVISIBILITY
}

\author{
D. D. ANDERSON
}

(Communicated by Louis J. Ratliff, Jr.)

\begin{abstract}
Let $D$ be an integral domain with integral closure $\bar{D}$. We show that the group of divisibility $G(D)$ of $D$ is finitely generated if and only if $G(\bar{D})$ is finitely generated and $\bar{D} /[D: \bar{D}]$ is finite. We also show that $G(D)$ is finitely generated if and only if the monoid of finitely generated fractional ideals of $D$ (under multiplication) is finitely generated.
\end{abstract}

Let $D$ be an integral domain with quotient field $K$. Let $K^{*}$ be the multiplicative group $K-\{0\}$ and $U(D)$ the group of units of $D$. The quotient group $G(D)=K^{*} / U(D)$, partially ordered by $a U(D) \leq b U(D) \Leftrightarrow a \mid b$ in $D$, is called the group of divisibility of $D$. Thus, the positive cone $G_{+}(D)$ is $D^{*} / U(D)=\{a U(D) \mid a \in D-\{0\}\}$. The group $G(D)$ is order isomorphic to the group $P(D)$ of nonzero principal fractional ideals of $D$ (under multiplication) ordered by reverse inclusion, while $G_{+}(D)$ is order isomorphic to the submonoid $I P(D)$ of $P(D)$ consisting of nonzero principal integral ideals. However, for the most part, we will be interested only in the underlying group structure of $G(D)$.

The purpose of this paper is to characterize integral domains with finitely generated groups of divisibility. Theorem 3 states that $G(D)$ is finitely generated if and only if $G(\bar{D})$ is finitely generated and $\bar{D} /[D: \bar{D}]$ is finite, where $\bar{D}$ is the integral closure of $D$ and $[D: \bar{D}]=\{d \in D \mid d \bar{D} \subseteq D\}$ is the conductor. Theorem 4 shows that an integral domain $D$ with $G(D)$ finitely generated may be realized as a composite or pullback of a very special type. We also show (Theorem 5) that $G(D)$ is finitely generated if and only if the monoid $F^{*}(D)$ of nonzero finitely generated fractional ideals of $D$ (under multiplication) is finitely generated.

The study of integral domains with finitely generated groups of divisibility was inaugurated by B. Glastad and J. L. Mott [2]. We collect in Theorem 0 results from [2] which will be used throughout this paper without further reference.

Received by the editors March 5, 1990 and, in revised form, May 24, 1990.

1980 Mathematics Subject Classification (1985 Revision). Primary 13A05, 13F05, 13 G05. 
Theorem 0 (B. Glastad and J. L. Mott). Let $D$ be an integral domain with $G(D)$ finitely generated.

(1) $\bar{D}$ is a Bézout domain with only finitely many prime ideals.

(2) $\bar{D}$ is a finitely generated $D$-module.

(3) $G(D) \cong G(\bar{D}) \oplus U(\bar{D}) / U(D)$, where $G(\bar{D})$ is free and $U(\bar{D}) / U(D)$ is finite.

(4) If $P$ is a prime ideal of $D$ with $D / P$ infinite, then $D_{P}=\bar{D}_{P}$ is a valuation domain.

Proof. (1) [2, Theorem 2.1].

(2) [2, Theorem 3.9].

(3) Consider the exact sequence $0 \rightarrow U(\bar{D}) / U(D) \rightarrow G(D) \rightarrow G(\bar{D}) \rightarrow 0$. Then $G(\bar{D})$ is finitely generated and is also torsion-free since $\bar{D}$ is integrally closed. Hence $G(\bar{D})$ is free. Therefore, the exact sequence splits. By [2, Theorem 3.9], $U(\bar{D}) / U(D)$ is finite.

(4) $[2$, Corollary 3.6].

Theorem 1. Let $D$ be an integral domain with $G(D)$ finitely generated. Then $\bar{D} /[D: \bar{D}]$ is finite.

Proof. Since $\bar{D}$ is a finitely generated $D$-module, it suffices to show that $D /$ $[D: \bar{D}]$ is finite. Also, since $D$ is semi-quasilocal, it suffices to show that $D_{M} /$ $[D: \bar{D}]_{M}$ is finite for each maximal ideal $M$ of $D$ containing $[D: \bar{D}]$. Because $\bar{D}$ is a finitely generated $D$-module, $[D: \bar{D}]_{M}=\left[D_{M}: \bar{D}_{M}\right]$. Thus, using the facts that $\bar{D}_{M}$ is the integral closure of $D_{M}$ and that $G\left(D_{M}\right)$ is finitely generated, we have to show only that if $(D, M)$ is a quasilocal domain with $G(D)$ finitely generated, then $D /[D: \bar{D}]$ is finite. If $D / M$ is infinite, then $D=\bar{D}$, and the result follows. So we may assume that $D / M$ is finite. Let $Q_{1}, \ldots, Q_{n}$ be the maximal ideals of $\bar{D}$. Then $\left(1+Q_{1} \cap \cdots \cap Q_{n}\right) /(1+M)$, being isomorphic to a subgroup of $U(\bar{D}) / U(D)$, is finite. Suppose that

$$
\left(1+Q_{1} \cap \cdots \cap Q_{n}\right) /(1+M)=\left\{\left(1+t_{1}\right)(1+M), \ldots,\left(1+t_{s}\right)(1+M)\right\} .
$$

For $q \in Q_{1} \cap \cdots \cap Q_{n}, 1+q=\left(1+t_{i}\right)(1+m)$ for some $i$ with $1 \leq i \leq s$ and $m \in M$. Thus $q=(1+m) t_{i}+m$, so $\bar{q}=(1+m) \bar{t}_{i}$ in $Q_{1} \cap \cdots \cap Q_{n} / M$. But $1+m$ is a unit, so $D \bar{q}=D \bar{t}_{i}$. Hence $Q_{1} \cap \cdots \cap Q_{n} / M$ has only finitely many cyclic $D$-submodules and hence has finite length as a $D$-module. Since $D / M$ is finite, $Q_{1} \cap \cdots \cap Q_{n} / M$ is actually finite. Now $\bar{D} / Q_{1} \cap \cdots \cap Q_{n} \cong \bar{D} / Q_{1} \times \cdots \times \bar{D} / Q_{n}$ is finite, since each $\bar{D} / Q_{i}$ is a finitely generated $D$-module. Thus $\bar{D} / M$ is finite, and hence so is $\bar{D} / D$. Therefore $D /[D: \bar{D}]=D / \operatorname{ann}(\bar{D} / D)$ is finite.

Thus $\bar{D} /[D: \bar{D}]$ is a finite principal ideal ring. Let $Q_{1}, \ldots, Q_{n}$ be the maximal ideals of $\bar{D}$ containing $[D: \bar{D}]$. Then $[D: \bar{D}]=B_{1} \cap \cdots \cap B_{n}$, where $B_{i}$ is $Q_{i}$-primary and $\bar{D} /[D: \bar{D}] \cong \bar{D} / B_{1} \times \cdots \times \bar{D} / B_{n}$, where $\bar{D} / B_{i}$ is a finite special principal ideal ring with finite residue field $\bar{D} / Q_{i}$. Since $\bar{D} / B_{i}$ is finite, either $Q_{i}$ is principal, in which case $B_{i}=Q_{i}^{n_{i}}$ for some $n_{i} \geq 1$, or $Q_{i}$ is not principal, and $B_{i}=Q_{i}$. 
The following lemma, while probably well known, is stated for the convenience of the reader:

Lemma 2. Let $D$ be a semi-quasilocal ring and $I$ an ideal of $D$. Let $S$ be a subring of $D / I$ and let $R=\pi^{-1}(S)$ where $\pi: D \rightarrow D / I$ is the natural map. Thus $U(D) / U(R) \cong U(D / I) / U(S)$.

Proof. Let $Q_{1}, \ldots, Q_{n}$ be the maximal ideals of $D$. Now the natural map gives a group homomorphism $U(D) \rightarrow U(D / I)$. Moreover, this map is surjective. Let $x+I \in U(D / I)$. Since $(x, I)=D \not \subset Q_{1} \cup \cdots \cup Q_{n}$, by [3, Theorem 124], there exists an $i \in I$ such that $x+i \notin Q_{1} \cup \cdots \cup Q_{n}$. Thus $x+i \in U(D)$, and $x+I=(x+i)+I$. Hence the map $U(D) \rightarrow U(D / I)$ is surjective. So the map $U(D) \rightarrow U(D / I) / U(S)$ is surjective and it has kernel $U(R)$. Thus $U(D) / U(R) \cong U(D / I) / U(S)$.

Theorem 3. For an integral domain $D$, the following conditions are equivalent.

(1) $G(D)$ is finitely generated.

(2) $G(\bar{D})$ is finitely generated, and $\bar{D} /[D: \bar{D}]$ is finite.

Proof. (1) $\Rightarrow(2)$. Suppose that $G(D)$ is finitely generated. Then $G(\bar{D})$, being a homomorphic image of $G(D)$, is also finitely generated. Theorem 1 gives the result that $\bar{D} /[D: \bar{D}]$ is finite.

$(2) \Rightarrow(1)$. Since $G(\bar{D})$ is finitely generated, $\bar{D}$ is a semi-quasilocal Bézout domain. Now $G(\bar{D})$ is a finitely generated free Abelian group, so the exact sequence $0 \rightarrow U(\bar{D}) / U(D) \rightarrow G(D) \rightarrow G(\bar{D}) \rightarrow 0$ splits. Hence $G(D) \cong$ $G(\bar{D}) \oplus U(\bar{D}) / U(D)$. Moreover, by Lemma 2 ,

$$
U(\bar{D}) / U(D) \cong U(\bar{D} /[D: \bar{D}]) / U(D /[D: \bar{D}])
$$

is finite. So $G(D)$ is finitely generated.

We next show that integral domains with finitely generated groups of divisibility may be realized as composites or pullbacks of a very special type.

Theorem 4. Let $D$ be an integral domain with $G(D)$ finitely generated. Then $\bar{D}$ is a Bézout domain with $G(\bar{D})$ a finitely generated free Abelian group, and $\bar{D} /[D: \bar{D}]$ is finite. Let $\pi: \bar{D} \rightarrow \bar{D} /[D: \bar{D}]$ be the natural map. Then $D=$ $\pi^{-1}(D /[D: \bar{D}])$. Conversely, suppose that $T$ is an integral domain with $G(T)$ finitely generated, and let $I$ be an ideal of $T$ with $T / I$ finite. Let $S$ be a subring of $T / I$. Then $D=\pi^{-1}(S)$, where $\pi: T \rightarrow T / I$ is the natural map, is an integral domain having the same quotient field as $T, I \subseteq[D: T], \bar{D}=\bar{T}$, and $G(D)$ is finitely generated. If $T$ is Bézout, $\bar{D}=T$ and $G(D) \cong G(T) \oplus U(T) / U(D) \cong$ $G(T) \oplus U(T / I) / U(S)$.

Proof. We have already proved the first part of Theorem 4. So suppose that $T$ is an integral domain with $G(T)$ finitely generated. Then $D$ is a subring of $T$ containing $I$. Hence $I \subseteq[D: T]$, and $D$ and $T$ have the same quotient field. Since $T / I$ is a finitely generated $S$-module, $T$ is a finitely generated $D$-module. Hence $\bar{D}=\bar{T}$. Lemma 2 gives the result that $U(T) / U(D) \cong U(T / I) / U(S)$ 
is finite. From the exact sequence $0 \rightarrow U(T) / U(D) \rightarrow G(D) \rightarrow G(T) \rightarrow 0$ with the two outside groups finitely generated, we see that $G(D)$ is also finitely generated. If $T$ is Bézout, then $\bar{D}=\bar{T}=T$, and $G(D) \cong G(\bar{D}) \oplus U(\bar{D}) / U(D) \cong$ $G(T) \oplus U(T / I) / U(S)$.

Let $D$ be an integral domain with quotient field $K$, and let $F^{*}(D)$ be the monoid of nonzero finitely generated fractional ideals of $D$ (under multiplication). Now if $F^{*}(D)$ is finitely generated, then the group $\operatorname{Inv}(D)$ of invertible ideals of $D$ is also finitely generated, being generated by those generators for $F^{*}(D)$ which are invertible. Hence the subgroup $P(D)$ of nonzero principal fractional ideals of $D$ is also finitely generated. Hence $G(D) \cong P(D)$ is finitely generated. (Since $D$ is semi-quasilocal, we actually have $\operatorname{Inv}(D)=P(D)$.) We will show that the converse is true, but we first consider the more general situation where $\bar{D}$ is assumed only to be Bézout.

Suppose that $D$ is an integral domain, with $\bar{D}$ being Bézout. The monoid homomorphism $F^{*}(D) \rightarrow P(\bar{D}) \cong G(\bar{D})$ given by $J \rightarrow J \bar{D}$ is surjective with "kernel" $B^{*}(D)=\left\{J \in F^{*}(D) \mid J \bar{D}=\bar{D}\right\}$. We have an exact monoid sequence $0 \rightarrow B^{*}(D) \rightarrow F^{*}(D) \rightarrow G(\bar{D}) \rightarrow 0$.

Suppose that, in addition to $\bar{D}$ being Bézout, the exact sequence $0 \rightarrow$ $U(\bar{D}) / U(D) \rightarrow G(D) \rightarrow G(\bar{D}) \rightarrow 0$ splits. (This is the case if $G(\bar{D})$ is finitely generated.) Let $g: G(\bar{D}) \rightarrow G(D)$ be a splitting. We may view $g$ as a map from $P(\bar{D})$ to $P(D)$. In this context, the fact that $g$ is a splitting amounts to saying that $g(\bar{D} x) \bar{D}=\bar{D} x$. Using $g$, we define the map $\theta: F^{*}(D) \rightarrow P(\bar{D}) \times B^{*}(D)$ by $\theta(J)=\left(J \bar{D},(g(J \bar{D}))^{-1} J\right)$. We next show that $\theta$ is a monoid isomorphism and that $B^{*}(D)$ is finite when $G(D)$ is finitely generated.

Theorem 5. Let $D$ be an integral domain such that $\bar{D}$ is a Bézout domain and such that the exact sequence $0 \rightarrow U(\bar{D}) / U(D) \rightarrow G(D) \rightarrow G(\bar{D}) \rightarrow 0$ splits. (This is the case if $G(D)$, or more generally $G(\bar{D})$, is finitely generated.) Then $F^{*}(D) \cong G(\bar{D}) \times B^{*}(D)$. If $G(D)$ is finitely generated, then $B^{*}(D)$ is finite. Thus $F^{*}(D)$ is finitely generated if and only if $G(D)$ is finitely generated.

Proof. We identify $G(\bar{D})$ with $P(\bar{D})$. We show that the previously defined map $\theta$ is a monoid isomorphism. Since $J \bar{D}=g(J \bar{D}) \bar{D}, g(J \bar{D})^{-1} J \bar{D}=\bar{D}$, so $\theta$ is well defined. The fact that $\theta$ is a monoid homomorphism easily follows from the fact that $g$ is a homomorphism. If $\theta(J)=\theta(K)$, then $J \bar{D}=K \bar{D}$, so $g(J \bar{D})=g(K \bar{D})$. Then $g(J \bar{D})^{-1} J=g(K \bar{D})^{-1} K$ implies that $J=K$. Finally, we show that $\theta$ is surjective. Let $(\bar{D} x, J) \in P(\bar{D}) \times B^{*}(D)$. So $J \bar{D}=\bar{D}$. Then

$$
\begin{aligned}
\theta(g(\bar{D} x) J) & =\left(g(\bar{D} x) J \bar{D},(g(g(\bar{D} x) J \bar{D}))^{-1} g(\bar{D} x) J\right) \\
& =\left(g(\bar{D} x) \bar{D},(g(g(\bar{D} x) \bar{D}))^{-1} g(\bar{D} x) J\right) \\
& =\left(\bar{D} x,(g(\bar{D} x))^{-1} g(\bar{D} x) J\right) \\
& =(\bar{D} x, D J)=(\bar{D} x, J) .
\end{aligned}
$$

Suppose that $G(D)$ is finitely generated. Let $J \in B^{*}(D)$. Then

$$
[D: \bar{D}]=[D: \bar{D}] \bar{D}=[D: \bar{D}](J \bar{D})=[D: \bar{D}] J \subseteq J \subseteq J \bar{D}=\bar{D} \text {. }
$$


So $[D: \bar{D}] \subseteq J \subseteq \bar{D}$. But by Theorem $1, \bar{D} /[D: \bar{D}]$ is finite. Hence $B^{*}(D)$ is finite.

Corollary 6. Let $D$ be an integral domain with $G(D)$ finitely generated. Then there exists a finite set $\left\{A_{1}, \ldots, A_{n}\right\}$ of finitely generated ideals of $D$ such that every nonzero finitely generated fractional ideal of $D$ has the form $x A_{i}$ where $x \in K^{*}$ and $1 \leq i \leq n$. Hence there exists a natural number $k$ such that every finitely generated fractional ideal of $D$ can be generated by $k$ elements.

Suppose that $D$ is an integral domain with $G(D)$ finitely generated. Then $B^{*}(D)$ is a finite monoid with identity $D$ and zero $\bar{D}$. The group of units of $B^{*}(D)$ is $P B^{*}(D)=\{D x \mid \bar{D} x=\bar{D}\}=\{D x \mid x \in U(D)\}$, which is naturally isomorphic to $U(\bar{D}) / U(D)$. Also, observe that a fractional ideal $A$ of $D$ is finitely generated if and only if $A \bar{D}$ is finitely generated. Certainly if $A$ is finitely generated, then $A \bar{D}$ is finitely generated. Conversely, suppose that $A \bar{D}$ is finitely generated, say $A \bar{D}=\bar{D} x$. Then $x^{-1} A \bar{D}=\bar{D}$. Choose $x_{1}, \ldots, x_{n} \in$ $x^{-1} A$ with $\left(x_{1}, \ldots, x_{n}\right) \bar{D}=\bar{D}$. So $\left(x_{1}, \ldots, x_{n}\right) \in B^{*}(D)$. Then, as in the proof of Theorem $5,[D: \bar{D}] \subseteq\left(x_{1}, \ldots, x_{n}\right) D \subseteq x^{-1} A \subseteq \bar{D}$. Since $\bar{D} /[D: \bar{D}]$ is finite, $x^{-1} A /\left(x_{1}, \ldots, x_{n}\right) D$ is finite. Hence $x^{-1} A$, and therefore $A$ itself, is finitely generated.

If $G_{+}(D)$ is finitely generated, then certainly $G(D)$ is finitely generated. The converse is false. Now, $G_{+}(D) \cong I P(D)$ is finitely generated if and only if $D$ has only a finite number of irreducible elements (up to units) and each nonzero nonunit element of $D$ is a product of irreducible elements. Such domains, called $C K$ domains in honor of Cohen and Kaplansky who first studied them, were the subject of [1]. It was shown [1, Theorem 4.5] that the following conditions on an integral domain $D$ are equivalent:

(1) $D$ is a CK domain,

(2) $G(D)$ is finitely generated and rank $G(D)=|\operatorname{Max}(D)|$ (we always have rank $G(D) \geq|\operatorname{Max}(D)|)$,

(3) $D$ is Noetherian, $G(D)$ is finitely generated, and $|\operatorname{Max}(D)|=$ $|\operatorname{Max}(\bar{D})|$,

(4) $\bar{D}$ is a semilocal PID with $D /[D: \bar{D}]$ finite and $|\operatorname{Max}(D)|=$ $|\operatorname{Max}(\bar{D})|$, and

(5) $D$ is a one-dimensional semilocal domain such that for each nonprincipal maximal ideal $M$ of $D, D / M$ is finite and $D_{M}$ is analytically irreducible.

In contrast, a Noetherian domain $D$ has $G(D)$ finitely generated if and only if $D$ is a one-dimensional semilocal domain such that for each nonprincipal maximal ideal $M, D / M$ is finite and $D_{M}$ is analytically unramified [1, Theorem 3.3]. It was observed [1, Corollary 3.6] that an integral domain $D$ is a local CK domain if and only if $G(D) \cong \mathbb{Z} \oplus F$, where $F$ is finite.

We end by examining integral domains $D$ with $G(D) \cong \mathbb{Z} \oplus \mathbb{Z} \oplus F$ where $F$ is finite. First, suppose that $D$ is an integral domain with $G(D) \cong \mathbb{Z} \oplus \mathbb{Z} \oplus F$, 
where $F$ is finite. Then $G(\bar{D}) \cong \mathbb{Z} \oplus \mathbb{Z}$, so $\bar{D}$ is a Bézout domain with at most two nonzero prime ideals. First, suppose that $\bar{D}$ has two maximal ideals. Then $\bar{D}$ is a semilocal PID with two maximal ideals. (In general, for a domain $D$ with $G(D)$ finitely generated, $\bar{D}$, or equivalently $D$, is Noetherian if and only if rank $G(D)=|\operatorname{Max}(\bar{D})|[1$, Corollary 3.5]). So either $D$ has two maximal ideals, in which case $D$ is a CK domain, or $D$ is a one-dimensional local domain with finite residue field. Both cases may occur. For let $K$ be a finite field and $T=K[X]_{(X)} \cap K[X]_{(X+1)}$ be a semilocal PID with two maximal ideals. Let $\pi: T \rightarrow K \oplus K$ be the natural map. If $k$ is a proper subfield of $K$, then $D=\pi^{-1}(k \oplus k)$ is a CK domain with two maximal ideals that is not a PID, and $G(D) \cong \mathbb{Z} \oplus \mathbb{Z} \oplus K^{*} / k^{*} \oplus K^{*} / k^{*}$. (Use Theorem 4 here and in the examples to follow.) If we take $K=\mathbb{Z} / 2 \mathbb{Z}$ and embed $K \rightarrow K \oplus K$ via the diagonal map, then $D=\pi^{-1}(K)$ is a local domain with $G(D) \cong \mathbb{Z} \oplus \mathbb{Z}$.

Second, suppose that $\bar{D}$ has only one maximal ideal $Q$. So $\bar{D}$ is a valuation domain. If $\operatorname{dim} \bar{D}=2$, then $\bar{D}$ must be a rank 2 discrete valuation domain. And either $D=\bar{D}$, or $\bar{D} / Q$ is finite and $D=\pi^{-1}(S)$, where $S$ is a subring of the finite SPIR $\bar{D} / Q^{n}$. For example, if $k \subset K$ is a pair of finite fields, then for $D=k[[X]]+K((X))[[Y]] Y, \bar{D}=K[[X]]+K((X))[[Y]] Y$ is a rank 2 discrete valuation domain and $G(D) \cong \mathbb{Z} \oplus \mathbb{Z} \oplus K^{*} / k^{*}$. Or, we may take $D=K\left[\left[X^{2}, X^{3}\right]\right]+K((X))[[Y]] Y$. Then $\bar{D}=K[[X]]+K((X))[[Y]] Y$ and $G(D) \cong \mathbb{Z} \oplus \mathbb{Z} \oplus K$, where here $K$ is the additive group of the field $K$. If $\operatorname{dim} \bar{D}=1$, then $(\bar{D}, Q)$ is a one-dimensional valuation domain with $G(\bar{D}) \cong$ $\mathbb{Z} \oplus \mathbb{Z}$. Since $\bar{D} /[D: \bar{D}]$ must be finite, either $D=\bar{D}$ or $[D: \bar{D}]=Q$ and $\bar{D} / Q$ is finite. Thus $D=\pi^{-1}(k)$, where $\pi: \bar{D} \rightarrow \bar{D} / Q$ is the natural map and $k$ is a subfield of the field $K=\bar{D} / Q$. Here $G(D) \cong \mathbb{Z} \oplus \mathbb{Z} \oplus K^{*} / k^{*}$.

\section{REFERENCES}

1. D. D. Anderson and J. L. Mott, Cohen-Kaplansky domains: integral domains with a finite number of irreducible elements, J. Algebra (to appear).

2. B. Glastad and J. L. Mott, Finitely generated groups of divisibility, Contemp. Math. 8 (1982), 231-247.

3. I. Kaplansky, Commutative rings, rev. ed., Univ. of Chicago Press, Chicago, 1974.

Department of Mathematics, The University of Iowa, Iowa City, Iowa 52242 